\title{
STABILITY OF A SWITCHED LINEAR SYSTEM
}

\section{At-Tasneem Mohd Amin ${ }^{1,2}$, Sallehuddin Mohamed Haris ${ }^{2}$ and Zulkifli Mohd Nopiah ${ }^{3}$}

${ }^{1}$ Faculty of Mechanical Engineering, Universiti Malaysia Pahang, 26600 Pekan, Pahang, Malaysia

Emil: tasneem@ump.edu.my

${ }^{2}$ Department of Mechanical and Material Engineering, Faculty of Engineering and the Built Environment,

Universiti Kebangsaan Malaysia, 43600 UKM Bangi, Malaysia

${ }^{3}$ Fundamental Engineering Unit, Faculty of Engineering and the Built Environment,

Universiti Kebangsaan Malaysia, 43600 UKM Bangi, Malaysia

\begin{abstract}
Hybrid systems are dynamic systems that arise out of the interaction of continuous state dynamics and discrete state dynamics. Switched systems, which are a type of hybrid system, have been given much attention by control systems research over the past decade. Problems with the controllability, observability, converseability and stabilizability of switched systems have always been discussed. In this paper, the trend in research regarding the stability of switched systems will be investigated. Then the variety of methods that have been discovered by researchers for stabilizing switched linear systems with arbitrary switching will be discussed in detail.
\end{abstract}

Keywords: Stability; Switched linear system; Lyapunov function.

\section{INTRODUCTION}

Switched systems are made up of a collection of linear subsystems with rules that govern the switching between these subsystems (Sun \& Ge, 2005). The switching law may be either supervised or unsupervised, and time-driven or event-driven (Ge, Zhendong, \& Lee, 2001). Switched systems exist in many practical applications, for example in control systems for gear transmissions, airplanes, traffic control and also for switching power in the electric industry (Liberzon, Hespanha, \& Morse, 1999). Much research has been done with regard to switched systems. These encompass the concept of system controllability, observability and also the stabilizability of a switched system.

Given a linear time invariant system (Gopal, 2003)

$$
\begin{gathered}
\dot{x}(t)=A x(t)+b u(t) \\
y(t)=c x(t)
\end{gathered}
$$

where $A, b$, and $c$ are a matrix $n \times n, n \times 1$, and $1 \times n$ respectively, $x(t)$ which $n \times 1$ is a state vector, $y(t)$ and $u(t)$ are the input and output of the system. The system is said to be controllable when input $u$ is able to transfer the initial state $x(0)$ to another state $x(t)$ in finite time. For a switched system, the system is said to be controllable if at the initial time and state a switching sequence exists that leads the state vector to the final state within a finite interval. The system is also said to be observable, given any 
switching sequence within a finite interval, in which the initial state can only be determined using the output vector. In other words, by utilizing the output vector of the system it is not impossible to determine the behavior of the whole system (Sun \& Ge, 2005). This state can be simplified to; if state vector $x \in R^{n}$ is controllable/ reachable/ unobservable at time $t_{0}$, then $x$ is controllable/reachable/unobservable at any arbitrary given instant of time.

Extensive research with regard to the controllability of continuous-time and discrete-time linear switched systems has been carried out by Ge, Zhendong, and Lee (2001) and Yijing, Guangming, and Long (2003) respectively for its geometric characterization. Even though this definition was constructed by only taking into consideration a single input, Guangming, Dazhong, and Long (2002) has however proven that the controllability of a multi-input system is equivalent to that of singleinput systems. It was later proved by Guangming and Long (2002) that for switched linear systems, a basic switching sequence exists such that the controllable state set of this basic switching sequence is equal to the controllable state set of the system. In contrast, a different emphasis of research was taken by $\mathrm{Vu}$ and Liberzon (2006), who were interested in a new issue in switched systems. They focused on the invertibility problem of a pair of subsystems for continuous-time linear switched systems. With information on the initial state and the output state, the switching signal and input state can be recovered. By introducing the concept of singular pairs for two systems in discrete and continuous states, an algorithm was presented for determining switching signals and inputs that generate a given output in a finite interval, when there is a finite number of such switching signals and inputs. However, this paper focuses on the stability of switched systems with subsystems which comprise of continuous-time linear systems. The discussion will also only take into account switched systems with arbitrary switching.

\section{STABILITY OF SWITCHED SYSTEMS}

Stability can be defined when all of the controllable state variables have stable dynamics, or if there are non-controllable state variables then all the state variables always remain within the boundaries of system behavior. In research on the analysis of the stability of switched systems, the scenario is that most researchers have the tendency to make conclusions on the behaviors of the switched systems without any theoretical application in finding the solutions of a hybrid system.

There are studies on the stability of switched systems that began with interpreting algorithms in the form of difference equations for continuous-time systems and differential equations for discrete-time systems (Brayton \& Tong, 1979, 1980). However, the main idea in the study of stability within hybrid systems is that when a Lyapunov function can be created from each subsystem, and by adjusting it with the switching mechanisms of the system, the stability of the switched system can be achieved. Extracting from Zhu, Cheng, and Qin (2007), and taking into account linear switched systems

$$
\dot{x}=A_{\sigma(t)} x
$$

where $\sigma(t):[0,+\infty) \rightarrow \Lambda$ is a continuous function with $\Lambda=\{1,2, \ldots, N\}$. 
To ensure the stability of the switched system in Eq. (1) with arbitrary switching, the common quadratic Lyapunov function (CQLF) is sufficient. The common quadratic Lyapunov function, $x^{T} P x$, with a positive definite matrix,$P>0$ is called the CQLF to $\left\{A_{\lambda} \mid \lambda \in \Lambda\right\}$ if

$$
P A_{\lambda}+A_{\lambda}^{T} P<0, \quad \forall \lambda \in \Lambda
$$

Referring to Lyapunov (1992), the Lyapunov stability or $x_{e}$ occurs when all solutions for a particular dynamic system that start near the equivalent point $x_{e}$ remain in that position of close proximity. The robustness of the stability is further increased when all the solutions starting near $x_{e}$ approach $x_{e}$, also known as being asymptomatically stable. This theorem is also supported by Martin and Dayawansa (1996) for switching within finitely linear systems with arbitrary switching, in which the asymptomatic stability for all switching paths is equivalent to the common Lyapunov function for all of the subsystems in the family. However, researchers face a difficulty in finding the upper bound of the degree of the system. This is following the expression of an example that shows that the Lyapunov function fails to form a CQLF. This theorem is also used by Jianhong, Xun, Yaping, and Guangfeng (2008) for linear timeinvariant systems, by using the LMI optimization approach to determine the CQLF. In addition to this discovery, King and Shorten (2004) stated that for a group of stable and finite matrices $\mathcal{A}=\left\{A_{1}, \ldots, A_{m}\right\}$, the CQLF will not exist if, and only if, all positive semidefinite matrices $X_{1}, \ldots, X_{m}$ do not equal zero, in which

$$
\sum_{i=1}^{m} A_{i} X_{i}+X_{i} A_{i}^{T}=0 .
$$

Research by Dayawansa and Martin (1999) on linear switched systems and Mancilla-Aguilar and Garcia (2000) on nonlinear switched systems with arbitrary switching have proved that each system respectively is globally uniformly asymptotically stable and locally uniformly exponentially stable with the converse Lyapunov theorem. Frequently it is seen that the focus of most studies is given to the quadratic stability of the system compared to uniformly asymptomatically stability. Quadratic stability can be achieved when the Lyapunov function is quadratic in the state variable and is independent of time. Nonetheless, the overall stability of the system is very dependent on the parameters and time. A study conducted by Mason, Sigalotti, and Daafouz (2007) found entire criterion of the stability of a system are actually equivalent, which can be rephrased as quadratic stability need only be tested on the quadratic polytopic Lyapunov function. They then found that this definition is not applicable for discrete time switched systems. This discovery has enforced the theorem proven by Sun (2007) for continuous-time switched systems, in which uniformly asymptomatically stability does not fulfill the quadratic Lyapunov function.

As an addition to the topic of stability behavior within switched systems, besides asymptomatic stability and quadratic stability, the input-to-state stability (ISS) is also an important property, though mainly for nonlinear systems. Due to the fact that the system might not overall be stable despite the stability of each subsystem, ISS becomes the preserver within the system in the efforts of achieving the overall stability of the whole system (Liberzon, Hespanha, \& Morse, 1999). To enforce this theory, Wenxiang, Changyun, and Zhengguo (2001) identified the type of mode of every subsystem before the suitable controller and the durations of controller usage were distinguished using model method to ensure that the whole system is in the ISS. Nesic and Liberzon (2005) 
then demonstrated the use of ISS small-gain theorems as a power extension to be used in hybrid systems. Further reference with regard to stability within systems with impulse effects can be found in Hespanha and Morse (2002), time-varying systems in Ezzine and Haddad (1988) and Qi, Guangming, and Long (2005), and time-delay in Dayawansa and Martin (1999). More information with regard to the analysis of stabilizability within switched systems in industries such as in the aircraft industry and the PI controllers of vehicles with automatic transmission can be found in Decarlo, Branicky, Pettersson, and Lennartson (2000), and in Brockett (1993) with regard to manual transmission.

\section{Multicontrollers}

In this method, the main aim is to construct a multicontroller system within a hybrid system. This multicontroller will be used and will effectively switch among the controllers for each subsystem, taking into account that a single controller is incapable of stabilizing the whole system with any switching sequence. Hespanha and Morse (2002) believe that if a multicontroller is chosen correctly then for every switching between subsystems it will be guaranteed to be uniformly exponentially stable. The system studied is the time invariant system. Each stable controller transfer matrix for every switching between subsystems will be mentioned and represented by equations in the form of Youla parameterization. This implies that switched systems will occur through certain parameters, compared to switching through a controller transfer matrix. The formation of multicontrollers in the form of the Youla parameterization must fulfill the Lyapunov function. This method of multicontrollers is also used by Stewart and Dumont (2006) for discrete time systems.

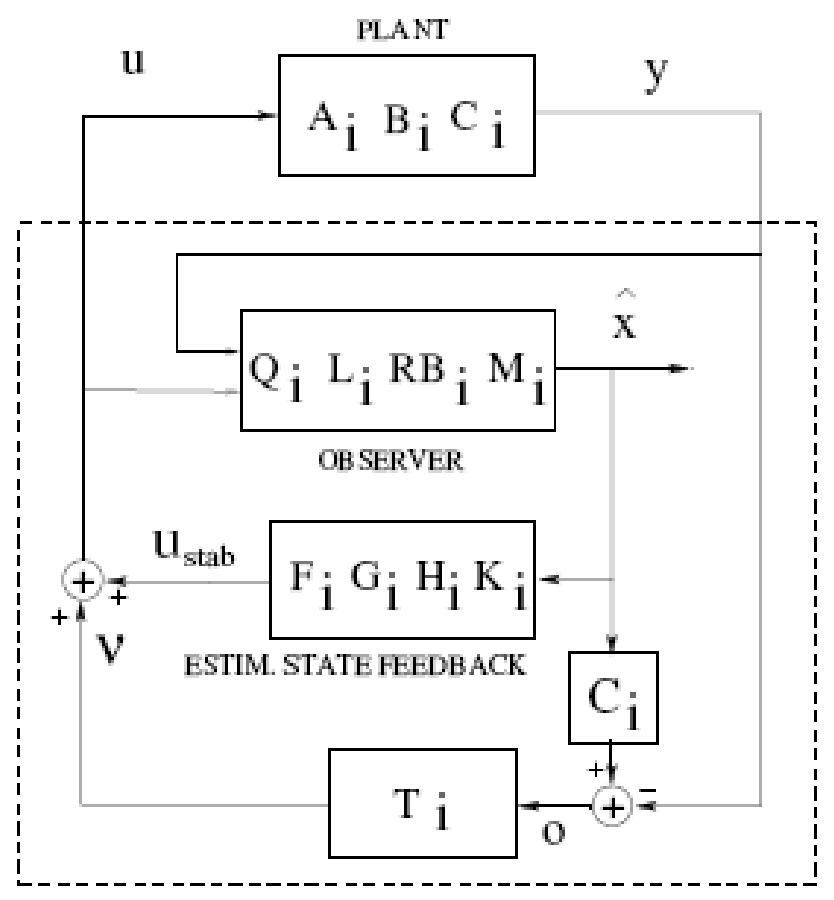

Figure 1. Switching compensator (Blanchini, Miani, \& Mesquine, 2008) 
Hespanha, Santesso, and Stewart (2007) raised a particular concern regarding the use of this technique: 'Is this technique applicable for use in switched systems that possess an interconnection between the feedback loop and the multicontrollers? If it is applicable, then how do we obtain the initial state with the controller after it has transformed back into the feedback loop?' Consequently, they introduced normconstraints in the optimization of the state-reset, in which a transient response will be produced which also preserves the (input to state) stability of the system. However, an argument raised by Blanchini et al. (2008) stated that the system that uses the Youla Parameter could not be arbitrary because it is already constructed. Therefore he introduce an extended controller device to the system with the Youla Parameter, called the switching compensator (consisting of an observer and a (dynamic) state feedback), as shown in Figure 1. This is supported by a polyhedral Lyapunov function based on the separation principle to fix the problem. However, all of them cannot provide the bounds for the system order, and it is highly computationally-demanding.

\section{Hurwitz Stability}

Referring to Eq. (2) with regard to guaranteeing the stability of switched systems within linear systems, the quest for finding the existence of a Lyapunov function is a conservative way of finding stability. Besides this method, Mason and Shorten (2003) conjectured that asymptomatic stability can be achieved in a positive linear system with arbitrary switching by testing the Hurwitz stability of the convex hull of a Metzler matrix set. The Hurwitz matrix is a square-structured matrix of $n \times n$ which is built together with a constant in a particular polynomial as follows

$$
H(p)=\left[\begin{array}{cccccc}
a_{1} & a_{3} & a_{5} & a_{7} & \cdots & 0 \\
a_{0} & a_{2} & a_{4} & a_{6} & \cdots & 0 \\
0 & a_{1} & a_{3} & a_{5} & \cdots & 0 \\
0 & a_{0} & a_{2} & a_{4} & \cdots & 0 \\
\vdots & \vdots & \vdots & \vdots & \ddots & 0 \\
0 & 0 & 0 & 0 & \cdots & 0
\end{array}\right]
$$

According to Zhang, Shen, and Han (2008), there are two criteria to test the stability of Hurwitz. One of the indirect methods is by testing the Eigenvalue of the matrix, including computing its Jordan canonical function, calculating the invariant factors, etc. However it is not an easy task to complete these computations due to computational complexity. Another method deals with stability, directly based on the entries of a given matrix. This conjecture can be true for a system which is only constructed from a pair of second order Metzler matrices, and for a system which is constructed from the arbitrary finite number of second order Metzler matrices, while the conjecture is generally false for higher order systems (Gurvits et al, 2007). This assumption is also taken up by Guisheng, Derong, Imae, and Kobayashi (2006) for continuous-time systems, forming an algorithm based on Lie Algebra for which the system is exponentially stable with arbitrary switching. 


\section{State Transformation}

The state variable transformation method is a way of changing the initial state variable of the system to a new state that is capable of creating a stabilizing strategy for that system before the new state is changed back to its initial state. Davrazos and Koussoulas (2002) utilized the canonical form in canonical coordinates as a medium for searching for the stabilizing strategy for switched linear systems. The problem of stability for this system is interpreted by using a state feedback control in canonical composition. Then, a state estimator is introduced within the system to estimate the state variable to be sent to the feedback loop and to achieve the algorithm of the overall system before it is changed back to its initial state. The main objectives are to find the suitable control input and switching laws which guarantee that the system will be uniformly asymptomatically stable. Before the transformation process it has to be ensured that the system is controllable and observable. The system is only stabilizable if the state space matrices are Hurwitz or equivalent, and the unstable mode of the state space matrices is controllable. Further reading with regard to state transformation can be found in Geng (2010).

\section{Linear State Transformation}

Alternatively, Li, Wen, and Soh (2001) introduced a linear state transformation to find a stable convex combination for a class of switched systems. The linear state transformation will decompose each subsystem into stable and unstable parts; in which for each stable part a Lyapunov function naturally exists. Under some conditions imposed on the original switched system, the sum of these Lyapunov functions is shown to be a Lyapunov function for a convex combination of the whole switched system.

\section{State-Switched Transformation Using Differential Petri Nets}

Differential Petri Nets (DPN) is a simulation software which is the sequel for another simulation software called "Hybrid Petri Nets", used for stabilizing switched linear systems (Davrazos \& Koussoulaz, 2007). Petri Nets is a basic hybrid system controller configuration to control continuous switching transformation from an initial discrete event to an output discrete event from the continuous input signal and output signal (Moor et al., 2006). The state transformation introduced in Petri Nets, exploits the capability and the advantages of continuous type Petri Net models in representing continuous varying quantities in a discrete event setting by making use of the simulation mechanism. The stability analysis by the novel transformation of the equation in the DPN of discrete event systems compared with the state switched system in a DPN framework using switching hyperplanes was presented. The stability condition was achieved and expressed in Linear Matrix Inequalities (LMI). The analysis of stability in state-switched hybrid systems with state transition using DPN has been divided into two parts. The first part is to model the state-switched hybrid system by switching hyperplanes in the DPN framework and the second part concerns transforming the DPN fundamental equation into a linear switched system form.

Figure 2 shows the model of the subsystem in DPN working plane form. Transition between the two different subsystems, expressed in the form of $i$ and $j$ as $f_{i j}^{T} x(t)=0$, is modelled in the DPN framework. The differential places $P_{D F 1}$ and $P_{D F 2}$ are tested for the expression $a x_{1}+b x_{2}=0$. Alternatively, Figure 3 shows an example 
of a DPN framework that has been formed into subsystems $i$ and $j$. Further reading on this method can be found in Davrazos and Koussoulas (2002).

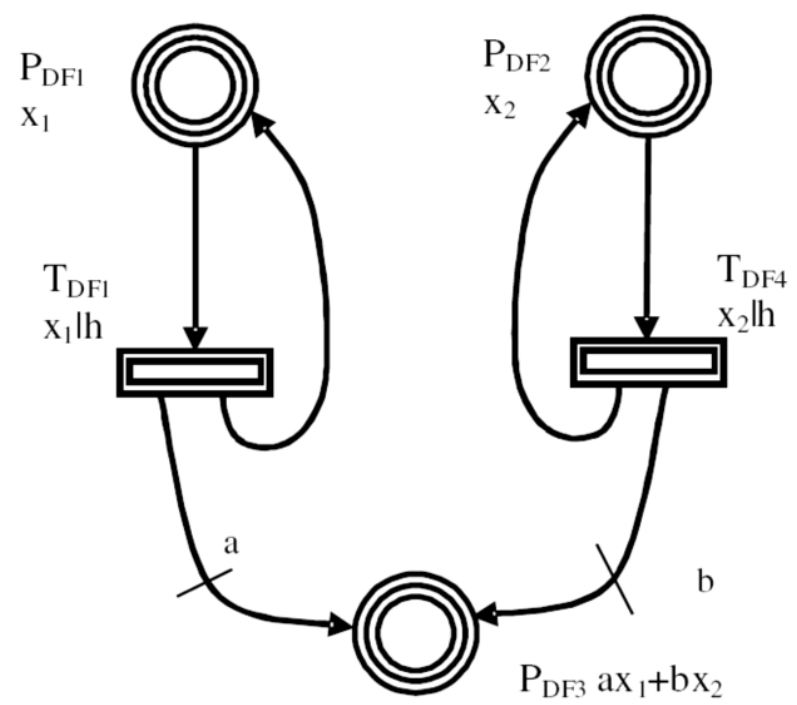

Figure 2. Modeling of hyperplanes in DPN framework (Davrazos \& Koussoulas, 2007)

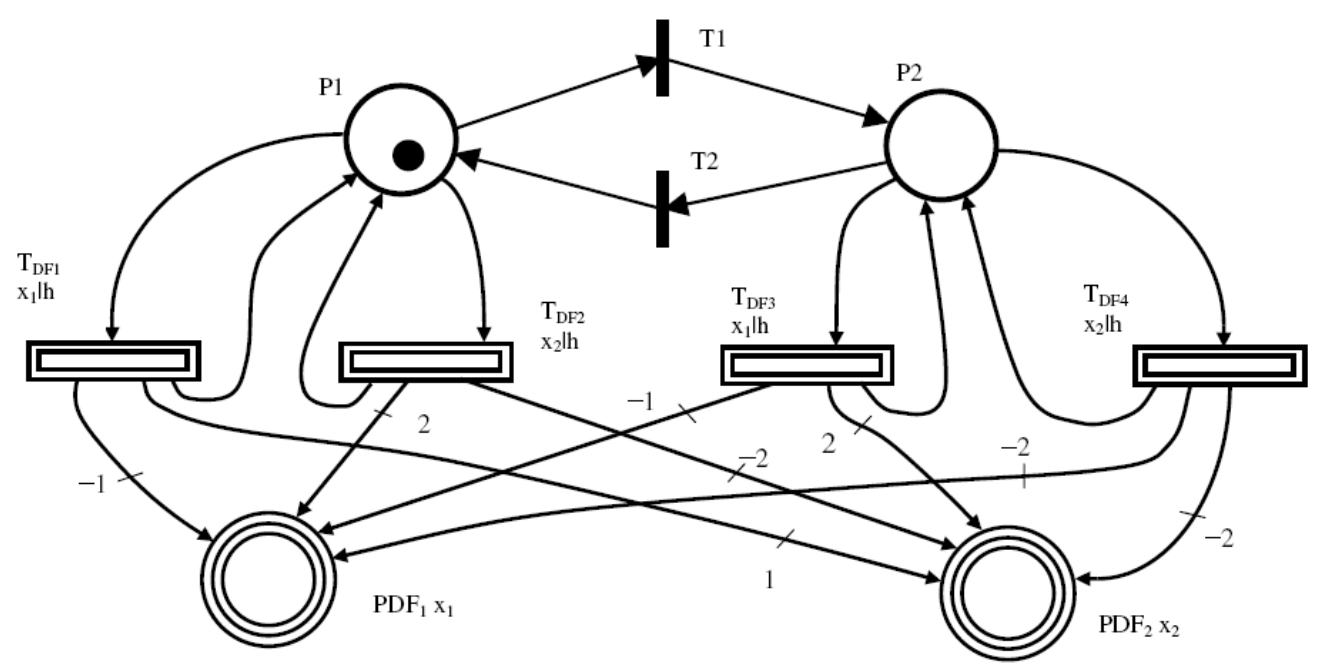

Figure 3. Representation of a switched system in a DPN framework (Davrazos \& Koussoulas, 2007)

\section{Stabilizing Switching Control Strategy}

Montagner, Leite, Oliveira, and Peres (2006), on the other hand, provided a convex design method for switching feedback gain for switched linear systems with arbitrary switching. A quadratic Lyapunov function with a common matrix is used to derive a stabilizing switching control strategy that, , for any arbitrary switching rule, guarantees: 
- The location of the poles of each linear subsystem of a continuous-time switched linear system is inside a chosen circle within the left-hand half of the complex plane.

- A minimum disturbance attenuation level for the closed-loop switched system.

The features mentioned above are said to be important since the first will improve the dynamic response assigning the bounds for the overshoot, settling time and frequency of oscillation. It also will be able to ensure the robustness of a switched system facing energy signal disturbances. The appropriate LMI design condition based on the quadratic Lyapunov functions with a common matrix with very low numerical complexity is presented. This LMI condition is allowed to determine the switched state feedback gains that stabilize the closed loop system, including the pole location and the robustness of the system. The stability of the closed-loop switched system with a $\gamma$ disturbance attenuation level and the pole location of each linear subsystem inside the circle $\mathcal{C}\left(d_{j}, r_{j}\right)$ is visualized in Figure 4:

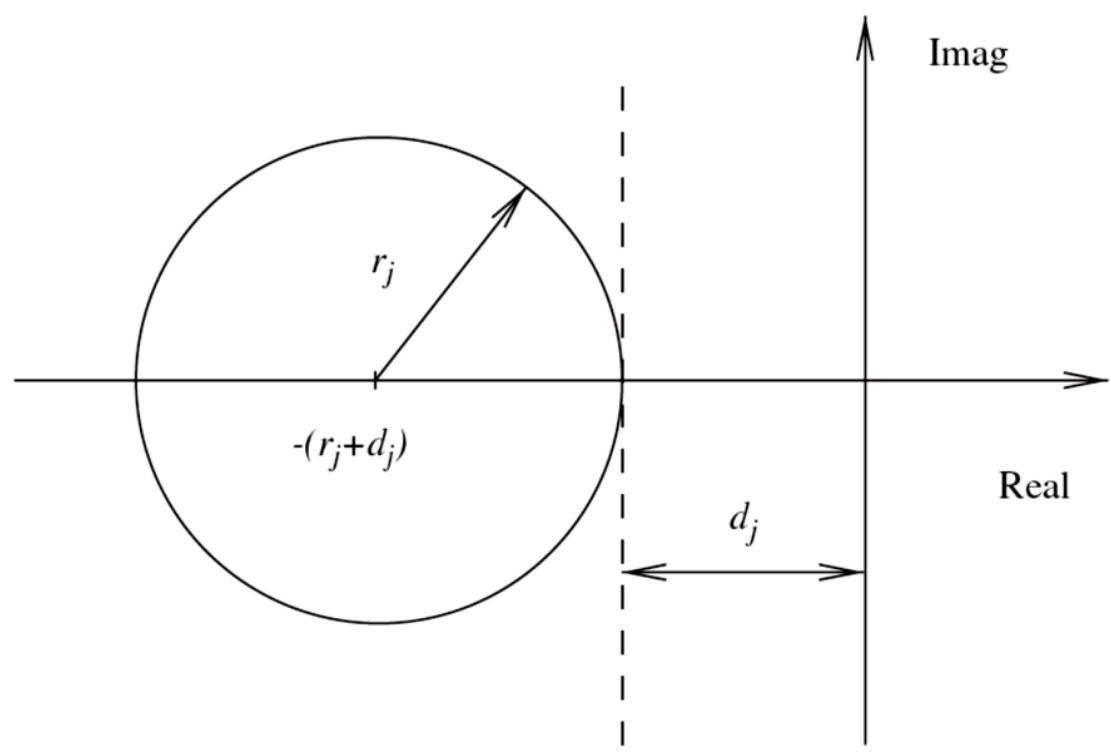

Figure 4. Circular region $\mathcal{C}\left(d_{j}, r_{j}\right)$ for pole location (Montagner et al., 2006)

\section{Lie Algebra}

Another popular method in the study of the stability of switched systems is the use of Lie Algebra, derived from a stable linear system. Lie Algebra is an algebraic structure that is usually used when learning of geometrical objects. Liberzon, Hespanha, and Morse (1999) conjectured that if the Lie Algebra generated from matrices of stable linear time invariant systems is nilpotent (which means that the Lie brackets of sufficiently high order equal zero), then the system is asymptotically stable for any switching signal. They have proved their conjecture to be true for two subsystems for third-order Lie brackets. The Lie bracket referred to here is the vector space in a particular graphic space in a binary operation. Further research has been carried out by Agrachev and Liberzon (1999), who discussed the subject by showing that an arbitrary switching system will be exponentially stable if the Lie algebra is solvable. Zhu, Cheng, and Qin (2007), however, claim that most methods for achieving stability using Lie 
Algebra are not solvable. Hence they proved that by combining Lie Algebra with the N$\mathrm{B}$ structure and also the CQLF, a new mathematical structure can be formed that offers a solution for the stability problem, which was unachievable through previous studies.

\section{Haris et al. (2007) Method}

This method was suggested to guarantee the quadratic stability of a switched system which consisted of second order subsystems. The aim is to find the existence of a set of feedback control laws $u_{i}=K_{i} x$ that share CQLF, $x^{T} P x$ in the subsystems. The subsystem is transformed to Brunovsky controllable canonical form to determine the existance of CQLF. If existing, it guarantees the stability of the switched system. The feedback controller, $K_{i}$ from the feedback control law can be found by solving two sets of linear inequalities (LI) in the original plane. The LI is $x \leq b$ where $x$ and $b$ are variables. The stability of a switched system is proven when the Lyapunov function in Eq. (3) is negative definite, which defines $P$ as a common Lyapunov function.

\section{DISCUSSION}

The stability of switched systems can be achieved by various methods. Among the approaches that can be used are mathematical and geometrical methods, the use of simulation software or the implementation of strategies across the system. Even though the method shown is focused on continuous linear systems with arbitrary switching, this method can be further explored in greater detail according to the type of system studied. Each system studied has been interpreted into mathematical equations or inequalities. The mathematical equations are then used to prove the algorithms created. A few questions do arise from this discussion. Will the equations and inequalities be solvable if the algorithms were later applied for use in industry and the field of education? Will computerized calculations be able to solve the problems faced? And what is the most suitable software to actually solve this problem?

The method using Youla parameterization is highly computationally demanding. Computing the Hurwitz stability by solving the Eigenvalues in the Jordan canonical function is complicated when using a computerized system. Hence it has been proved by Zhang, Shen, and Han (2008) that it failed when used for higher order systems. Furthermore, determining the stability using the state transformation method by Davrazos and Koussoulas (2002) can only be solved if the state matrix is Hatwitz. Most Lie Algebra cannot be solved unless combined with N-B structures and CQLF. On the other hand, Montagner et al. (2006) used simple mathematics to determine the CQLF that guaranteed the stability of a switched system.

\section{CONCLUSION}

To summarize, most of the suggested method are actually very computationally demanding for solving the problems. As a solution to this problem, research is needed to test the computational demand when determining the feedback controller that guarantees the stability of a hybrid system. A new algorithm needs to be introduced which it is guaranteed to be less computationally demanding. 


\section{REFERENCES}

Blanchini, F., Miani, S., \& Mesquine, F. (2008). A separation principle for linear switching systems and parametrization of all stabilizing controllers. $47^{\text {th }}$ IEEE Conference on Decision and Control, pp. 953-958.

Brayton, R. K., \& Tong, C. H. (1979). Stability of dynamical systems: a constructive approach. IEEE Transactions on Circuits and Systems, 26, 224-234.

Brayton, R. K., \& Tong, C. H. (1980). Constructive stability and asymptotic stability of dynamical systems. IEEE Transactions on Circuits and Systems, 27, 1121-1130.

Brockett, R. W. (1993). Hybrid models for motion control systems. In: Trentelman, H. L. \& Willems, J. C. (eds.) Essays on control: perspectives in the theory and its applications, Cambridge, MA, Birkhäuser Boston, pp. 29-53.

Davrazos, G., \& Koussoulas, N. T. (2002). A general methodology for stability analysis of differential petri nets. Proceedings of the 10th Mediterranean Conference on Control and Automation, pp. 1-7.

Dayawansa, W. P., \& Martin, C. F. (1999). A converse Lyapunov theorem for a class of dynamical systems which undergo switching. IEEE Transactions on Automatic Control, 44 (4), 751-760.

Decarlo, R. A., Branicky, M. S., Pettersson, S., \& Lennartson, B. (2000). Perspectives and results on the stability and stabilizability of hybrid systems. Proceedings of the IEEE, 88 (7), 1069-1082.

Ezzine, J., \& Haddad, A. H. (1988). On the controllability and observability of hybrid systems. American Control Conference, pp. 41-46.

Ge, S. S., Zhendong, S., \& Lee, T. H. (2001). Reachability and controllability of switched linear systems. Proceedings of the 2001 American Control Conference, pp. 1898-1903.

Geng, Z. (2010). Switched stability design on canonical forms. IEEE International Conference on Information and Automation (ICIA), pp. 289-293.

Gopal, M. (2003). Control systems principles and design. New Delhi, McGraw Hill.

Guangming, X., Dazhong, Z., \& Long, W. (2002). Controllability of switched linear systems. IEEE Transactions on Automatic Control, 47 (8), 1401-1405.

Guangming, X., \& Long, W. (2002). Necessary and sufficient conditions for controllability of switched linear systems. Proceedings of the 2002 American Control Conference, pp. 1897-1902.

Guisheng, Z., Derong, L., Imae, J., \& Kobayashi, T. (2006). Lie algebraic stability analysis for switched systems with continuous-time and discrete-time subsystems. IEEE Transactions on Circuits and Systems II: Express Briefs, 53 (2), 152-156.

Hespanha, J. P., \& Morse, A. S. (2002). Switching between stabilizing controllers. Automatica, 38 (11), 1905-1917.

Hespanha, J. P., Santesso, P., \& Stewart, G. (2007). Optimal controller initialization for switching between stabilizing controllers. 46th IEEE Conference on Decision and Control, pp. 5634-5639.

Jianhong, W., Xun, L., Yaping, G., \& Guangfeng, J. (2008). An LMI optimization approach to Lyapunov stability analysis for linear time-invariant systems. Chinese Control and Decision Conference, pp. 3044-3048.

King, C., \& Shorten, R. (2004). A singularity test for the existence of common quadratic Lyapunov functions for pairs of stable LTI systems. Proceedings of the 2004 American Control Conference, pp. 3881-3884. 
Li, Z. G., Wen, C. Y., \& Soh, Y. C. (2001). Stabilization of a class of switched systems via designing switching laws. IEEE Transactions on Automatic Control, 46 (4), 665-670.

Liberzon, D., Hespanha, J. P., \& Morse, A. S. (1999). Stability of switched systems: a lie-algebraic condition. Systems and Control Letters, 37 (3), 117-122.

Lyapunov, A. M. (1992). General problem of the stability of motion. Bristol, PA, Taylor and Francis.

Mancilla-Aguilar, J. L., \& García, R. A. (2000). A converse Lyapunov theorem for nonlinear switched systems. Systems and Control Letters, 41 (1), 67-71.

Martin, C. F., \& Dayawansa, W. P. (1996). On the existence of a Lyapunov function for a family of switching systems. Proceedings of the 35th IEEE Decision and Control, pp. 1820-1823.

Mason, O., \& Shorten, R. (2003). A conjecture on the existence of common quadratic Lyapunov functions for positive linear systems. American Control Conference, pp. 4469-4470.

Mason, P., Sigalotti, M., \& Daafouz, J. (2007). On stability analysis of linear discretetime switched systems using quadratic Lyapunov functions. 46th IEEE Conference on Decision and Control, pp. 5629-5633.

Montagner, V. F., Leite, V. J. S., Oliveira, R. C. L. F., \& Peres, P. L. D. (2006). State feedback control of switched linear systems: an LMI approach. Journal of Computational and Applied Mathematics, 194(2), 192-206.

Nesic, D., \& Liberzon, D. (2005). A small-gain approach to stability analysis of hybrid systems. 44th IEEE Conference on Decision and Control and 2005 European Control Conference, pp. 5409-5414.

Qi, F., Guangming, X., \& Long, W. (2005). Stability analysis and stabilization synthesis for periodically switched linear systems with uncertaintie. American Control Conference, pp. 30-35.

Stewart, G. E., \& Dumont, G. A. (2006). Finite horizon based switching between stabilizing controllers. American Control Conference, pp. 1550-1556.

Sun, Z. (2007). Converse Lyapunov theorem for switched stability of switched linear systems. Chinese Control and Decision Conference, pp. 678-680.

Sun, Z., \& Ge, S. S. (2005). Analysis and synthesis of switched linear control systems. Automatica, 41, 181-195.

Vu, L., \& Liberzon, D. (2006). On invertibility of switched linear systems. 45th IEEE Conference on Decision and Control, pp. 4081-4086.

Wenxiang, X., Changyun, W., \& Zhengguo, L. (2001). Input-to-state stabilization of switched nonlinear systems. IEEE Transactions on Automatic Control, 46 (7), 1111-1116.

Yijing, W., Guangming, X., \& Long, W. (2003). Reachability and controllability of switched linear systems with state jumps. IEEE International Conference on Systems, Man and Cybernetics, pp. 672-677.

Zhang, W., Shen, S. Q., \& Han, Z. Z. (2008). Sufficient conditions for Hurwitz stability of matrices. Latin American Applied Research, 38, 253-258.

Zhu, Y. H., Cheng, D. Z., \& Qin, H. S. (2007). Constructing common quadratic Lyapunov functions for a class of stable matrices. Acta Automatica Sinica, 33(2), 202-204. 